\title{
Influencia de la Antigüedad y del total de Horas de Investigación en la Producción Científica de Docentes Universitarios
}

\author{
Olga G. Barbón*, Santiago F. Barriga, Amparo L. Cazorla y Lexinton G. Cepeda \\ Universidad Nacional de Chimborazo (UNACH), Facultad de Ciencias de la Educación, Humanas y \\ Tecnologías, Ave 10 de agosto y Eloy Alfaro, Riobamba, Ecuador. \\ (e-mail: olgagloria29@yahoo.com ogbarbon@unach.edu.ec) \\ * Autor a quien debe ser dirigida la correspondencia
}

Recibido Ago. 28, 2017; Aceptado Nov. 6, 2017; Versión final Mar. 5, 2018, Publicado Ago. 2018

\begin{abstract}
Resumen
Se analiza, mediante un estudio longitudinal, la producción científica en docentes universitarios de la Facultad de Ciencias de la Educación, Humanas y Tecnologías de la Universidad Nacional de Chimborazo (UNACH), en Ecuador. Especial atención se presta a la relación entre la experiencia en la docencia (antigüedad) y al total de horas dedicadas a la investigación. La recogida de datos se realizó entre junio del 2015 y junio del 2017. Se efectuaron análisis descriptivos y correlacionales entre las variables recogidas. Se llevaron a cabo análisis multivariados de varianza para examinar las relaciones bivariantes existentes entre las variables dependientes: experiencia en la docencia universitaria y el total de horas dedicadas a la investigación y la variable independiente, producción científica. Además, se exploró su capacidad predictiva sobre la producción científica, a través del análisis de regresión lineal múltiple. Este trabajo constituye un primer acercamiento a trazar el perfil general del investigador idóneo y de los factores que lo afectan. Se constató que el personal académico con un mayor número de publicaciones, tiene alrededor de 15 y 30 horas semanales asignadas para proyectos de investigación y producción científica.
\end{abstract}

\section{Influence of Seniority and total Research Hours on Scientific Production of University Professors}

\begin{abstract}
Through a longitudinal study, the scientific production in university professors of the Faculty of Educational Sciences, Humanities and Technologies of the Universidad Nacional de Chimborazo (UNACH), in Ecuador is analyzed. Special attention is paid to the relation between the teaching experience (seniority) and the total hours dedicated to research. The data collection was carried out between June 2015 and June 2017. Descriptive and correlational analyzes were carried out among the chosen variables. Multivariate analysis of variance was carried out to examine the bivariate relationships between the dependent variables: experience in university teaching and total hours dedicated to research and the independent variable, scientific production. In addition, the predictive capacity of the model on scientific production was explored through multiple linear regression analysis. This work is a preliminary approach to trace the ideal researcher profile and the factors that affect it. It was found that academic staff with higher number of publications, has about 15 and 30 hours assigned to research projects and scientific production.
\end{abstract}

Keywords: seniority in university; research hours; scientific production; university professors 


\section{INTRODUCCIÓN}

La producción científica de los docentes universitarios da cuenta de los resultados de las investigaciones, tanto básicas como aplicadas, que se desarrollan en este ámbito, convirtiéndose en una provechosa herramienta para el perfeccionamiento de la calidad académica de las Instituciones de Educación Superior (IES) a nivel mundial (Jiménez y Medina, 2016). En las últimas décadas ha habido un notable crecimiento del interés por este tema, probablemente asociado al impulso significativo que ha recibido la investigación académica, a su vez sustentado en la propia misión eminentemente social de las universidades; con lo cual resulta cada vez más necesario que su producción científica y los factores que intervienen en la misma sean objeto de reflexión y de análisis. (Aranguren, et al. 2016)

Por consiguiente, esta situación ha dado pie a que en las universidades se instaure una cierta "cultura de la producción científica" que amenaza con tomar el lugar de la "cultura de investigación". Tal afirmación no es menos válida en las universidades ecuatorianas, en las cuales se ha otorgado un gran peso a las publicaciones del personal académico en los criterios de evaluación y de acreditación de las mismas. Sin embargo, en la práctica, este proceso ha sido objeto de considerables obstáculos de naturaleza tanto individual (escaso desarrollo de habilidades investigativas en los docentes, insuficiente tiempo dedicado a la investigación, falta de motivación y de idoneidad, entre otros); como grupal (poco reconocimiento al papel de la publicación científica en el proceso investigativo, insuficientes competencias, recursos, tiempo y oportunidades para gestionar, producir y comunicar los resultados investigativos del grupo, falta de liderazgo y de cohesión en el grupo, entre otros) e institucional (presupuesto limitado para las actividades de investigación, falta de políticas de investigación claras, falta de reconocimiento y escasos incentivos a los investigadores, entre otros). A pesar de los factores anteriormente mencionados, fundamentalmente la escasez de horas asignadas a la investigación atenta contra la producción científica de los docentes universitarios. Diversos estudios han señalado lo extendido de este fenómeno, común a varios contextos, aunque sin respaldarlo con evidencia empírica. (Parra, 2007; Rabossi, 2014; Abramo et al., 2014; Romero y Pastor, 2012; Herrero, 2015; Farcas et al., 2017; Franzoni, y Sauermann, 2014; Rossi, y Rosli, 2015; Kemmis, et al., 2014; Simonneaux, 2014; Osborne, 2014; Schubert, y Yang, 2016).

Esta problemática no es ajena a la Universidad Nacional de Chimborazo (UNACH), la cual, paulatinamente, ha ido formulando visiones que han probado tener validez respecto al rol central de la producción científica en la generación de nuevos conocimientos. Aunado a ello, la necesidad de incrementar la producción científica de su planta académica ha promovido el desarrollo y la consolidación de pequeños grupos de investigadores. A pesar de esto, en el marco de los estudios sobre estos grupos, no han sido tenidos en cuenta con anterioridad algunos elementos participantes en el rendimiento de los mismos y, bajo esta perspectiva, quedan temas aún poco explorados. Uno de los vacíos existentes en este ámbito de investigación es la escasez de estudios en los que se haya analizado, de un modo específico, la producción científica de los docentes universitarios según su experiencia en la docencia de este nivel y el total de horas dedicadas a la investigación (Weissmann, 2014) De hecho, la mayoría de los trabajos publicados que se han aproximado empíricamente a la producción científica de este sector se han centrado únicamente en la calidad de los productos y su proceso de obtención (Álvarez y Juncosa, 2014; Barbón et al., 2017) así como en la influencia de la estructura y el funcionamiento de los grupos de investigación en su rendimiento científico (Rey et al., 2010; Cabezas et al., 2013), pero no han diferenciado entre dos antecedentes medulares: experiencia en la docencia universitaria y cantidad de horas dedicadas a la investigación per se, como factores determinantes del mismo.

En definitiva, se sigue echando en falta el estudio de esta arista de tan importante temática, la cual, si fuese analizada rigurosamente, podría predecir el éxito o el fracaso de diferentes políticas, formas de organización y planificación científico-académicas. Es por esto que resulta valioso descubrir los efectos específicos de estos antecedentes en la producción científica de los docentes universitarios, con lo cual se contribuye no solo a la identificación de aquellas variables y relaciones que permiten explicarla, dejando en claro en qué medida la carga horaria de investigación con la que cuentan estos sujetos está respondiendo al llamamiento internacional y nacional a la publicación científica, sino también al desarrollo científico, al ejercer una función orientadora en el diseño de políticas y de estrategias para gestionar la investigación a corto, mediano y largo plazo, aprovechando el recurso organizativo de los grupos de investigación.

Es válido resaltar que dentro de los resultados científicos que aportan los investigadores de la UNACH, los de carácter educativo, en particular, poseen elevada pertinencia por constituir en sí mismos propuestas para el mejoramiento de la calidad académica o reflexiones críticas sobre las diversas aristas del complejo proceso de formación de profesionales. En este sentido, puede afirmarse que la investigación que tiene lugar en la más antigua de las facultades de este prestigioso enclave universitario -la Facultad de Ciencias de la Educación, Humanas y Tecnologías (FCEHyT)- constituye un hito importante, que repercute positivamente en el fortalecimiento de una verdadera cultura investigativa en los docentes de la universidad en general. 
Con base en lo mencionado hasta aquí, el objetivo de este trabajo es comparar, mediante un estudio longitudinal, la producción científica en docentes universitarios de la FCEHyT según su experiencia en la docencia de este nivel y el total de horas dedicadas a la investigación; así como examinar las relaciones existentes entre la experiencia en la docencia universitaria, el total de horas dedicadas a la investigación y el género.

\section{METODOLOGÍA}

Durante el período 2015-2017, se llevó a cabo un estudio cuantitativo, descriptivo y correlacional en la Facultad de Ciencias de la Educación, Humanas y Tecnologías (FCEHyT) de la Universidad Nacional de Chimborazo (UNACH). Los participantes forman parte de una investigación longitudinal más amplia sobre la producción científica de los docentes universitarios, realizada en las universidades de la zona 3 del Ecuador.

Tuvieron lugar varios momentos - 6 fases- a lo largo del proceso investigativo, que se describen a continuación. Tras un primer momento de planificación y coordinación, durante el cual se procedió a la búsqueda bibliográfica exhaustiva y a la operacionalización de variables, se diseñó el instrumento de recogida de datos (fase 1). Con la primera versión del cuestionario se realizó una prueba piloto, que permitió determinar su consistencia interna, medida a través del coeficiente Alpha de Cronbach $(\alpha=0.87)$. Una segunda versión del cuestionario fue sometida al juicio de expertos, para ello se organizó un panel de cinco expertos que identificó los elementos que debían modificarse, respecto a la redacción de los ítems para garantizar la validez de contenido de los mismos (fase 2). La recogida de datos se extendió por dos años, entre junio 2015 y junio del 2017. A finales del mes de junio del año 2015 se realizó la primera recogida de información de los sujetos muestreados, mediante la técnica de muestreo casual (fase 3). Se tomaron datos de 37 docentes, con un rango de edad entre los 32 y 60 años (M: 44.72; DT: 8.820), directamente de los registros de investigación de la FCEHyT.

El 27 de abril del 2017 se concretó el primer Jueves Científico del nuevo ciclo académico. La actividad, de apoyo a la promoción de una cultura científica entre los docentes, incluyó el desarrollo de conferencias y conversatorios. En este marco se solicitó a las autoridades y a los docentes su autorización para la participación en el estudio. Todos los cuestionarios fueron auto administrados al ser enviados por correo electrónico y, una vez completados por los encuestados, fueron devueltos por el mismo medio. Previamente, a los docentes se les indicó que el objetivo del estudio era conocer el impacto de algunos aspectos en la producción científica del personal académico. Se les informó del anonimato de sus respuestas y se les insistió en la importancia de su sinceridad (fase 4). Con el propósito de atenuar el sesgo de selección forzada por categorías insuficientes y considerando que, si bien se preservó el anonimato, los cuestionarios incluían un código para identificar que pertenecían al mismo docente, en el mes de junio del 2017 se volvió a contactar a los muestreados para solicitarles que actualizaran sus datos en los cuestionarios enviados, en caso de que existiera alguna variación en su producción científica (fase 5). Ocho (8) de los sujetos se rehusaron a continuar en el estudio a causa de la excesiva saturación de tareas relacionadas con la cercanía del final del semestre. La muestra final quedó conformada por 25 docentes, de los cuales el 48\% pertenecían al sexo masculino (12) y el $52 \%$ al sexo femenino (13). Se desestimaron cuatro (4) cuestionarios en los que el porcentaje de pérdida de datos alcanzaba o superaba el $20 \%$.

Los datos obtenidos fueron almacenados en una base de datos de Microsoft Excel 2010, confeccionada al efecto, posteriormente fueron procesados estadísticamente a través de los paquetes estadísticos: SPSS, versión 21.0 y el programa AMOS 5.0. (fase 6). Se realizaron análisis descriptivos y correlacionales entre las variables: experiencia en la docencia universitaria, total de horas dedicadas a la investigación y los componentes de la producción científica (Tabla 1). Se llevaron a cabo análisis multivariados de varianza (MANOVA) para examinar las relaciones bivariadas existentes entre las variables, tomando como variables dependientes la experiencia en la docencia universitaria y el total de horas dedicadas a la investigación y como variable independiente el género (Tabla 2). Además, se exploró la capacidad predictiva del género, la edad, las horas dedicadas a la investigación y la experiencia docente sobre la producción científica, a través del análisis de regresión lineal múltiple. En todas las pruebas estadísticas se utilizó un nivel de significación de 0.05. (Tabla 3)

\section{RESULTADOS}

A continuación, se presentan los principales resultados del estudio realizado.

La Tabla 1 muestra los análisis descriptivos y las correlaciones entre las variables experiencia docente, horas dedicadas a la investigación, los componentes de la producción científica y edad de los participantes. Puede apreciarse que la experiencia docente se relacionó de forma positiva y muy significativa con las horas dedicadas a la investigación $(r=0.651 ; p<0.01)$, mientras que se relacionó de forma significativa con la 
producción de artículos científicos $(r=0.412 ; p<0.05)$, la producción de ponencias $(r=0.415 ; p<0.05)$ y la edad $(r=0.421 ; p<0.05)$. Por otro lado, las horas dedicadas a la investigación también se relacionaron positiva y muy significativamente con la producción de artículos científicos $(r=0.614 ; p<0.01)$ y de forma significativa con la producción de ponencias $(r=0.598 ; p<0.05)$ y la edad $(r=0.427 ; p<0.05)$. La producción de capítulos de libros se relacionó positivamente con la producción de artículos científicos y esta relación fue significativa ( $r=0.452$; $\mathrm{p}<0.05)$. Por otra parte, la producción de artículos científicos se relacionó, además, con la producción de ponencias de forma positiva y significativa $(r=0.494 ; p<0.05)$. La edad también se relacionó positiva y significativamente con la producción de ponencias $(r=0.510 ; p<0.05)$. Con base en esos resultados puede verse que al aumentar la experiencia docente aumentan las horas dedicadas a la investigación, la producción de artículos científicos y de ponencias y viceversa, es decir, al disminuir la experiencia docente disminuyen las horas dedicadas a la investigación, la producción de artículos científicos y la producción de ponencias. De igual modo, al aumentar la edad, también lo hace la experiencia docente, las horas dedicadas a la investigación y la producción de ponencias. Además, cuando aumentan las horas dedicadas a la investigación se incrementa la producción de artículos científicos y de ponencias.

Tabla 1: Estadísticos descriptivos y correlaciones entre las variables de estudio. ${ }^{*} p<0.05,{ }^{* *} p<0.01$

\begin{tabular}{|l|l|l|l|l|l|l|}
\hline & 1 & 2 & 3 & 4 & 5 & 6 \\
\hline 1-Experiencia docente & & & & & & \\
\hline 2-Horas dedicadas a la investigación & $0.651^{* *}$ & & & & & \\
\hline 3-Producción de capítulos de libros & 0.296 & 0.143 & & & & \\
\hline 4-Producción de artículos científicos & $0.412^{*}$ & $0.614^{* *}$ & $0.452^{*}$ & & & \\
\hline 5-Producción de ponencias & $0.415^{*}$ & $0.598^{*}$ & 0.064 & $0.494^{*}$ & & \\
\hline 6-Edad & $0.421^{*}$ & $0.427^{*}$ & 0.130 & 0.054 & $0.510^{*}$ & \\
\hline & & & & & & \\
\hline Media & 13.44 & 19.14 & 1.52 & 4.37 & 7.20 & 44.72 \\
\hline & & & & & & \\
\hline Desviación estándar & 4.65 & 9.86 & 0.84 & 3.64 & 9.15 & 7.43 \\
\hline
\end{tabular}

En el análisis multivariado de varianza (MANOVA), que empleó como variable independiente el sexo, ésta resultó ser no significativa (Lambda de Wilks=0.899, $F=0.336, p=0.909$ ). Puede verse en la Tabla 2 que no hubo diferencias entre los sexos en las variables analizadas. Esto quiere decir que el pertenecer a uno u otro género no influyó en las horas dedicadas a la investigación, tampoco en la producción de capítulos de libros, de artículos científicos y ponencias.

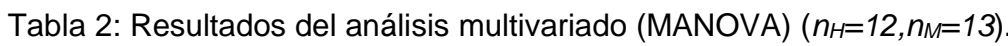

\begin{tabular}{|c|c|c|c|c|}
\hline \multicolumn{2}{|l|}{ Grupos } & \multirow{2}{*}{$\begin{array}{c}M(D E) \\
13.33(9.149)\end{array}$} & $F$ & $p$ \\
\hline Años de experiencia docente & Hombres & & \multirow{2}{*}{0.531} & \multirow{2}{*}{0.474} \\
\hline & Mujeres & $11.31(3.966)$ & & \\
\hline \multirow[t]{2}{*}{ Horas dedicadas a la investigación } & Hombres & $15.33(2.211)$ & \multirow{2}{*}{0.166} & \multirow{2}{*}{0.687} \\
\hline & Mujeres & $9.85(4.997)$ & & \\
\hline \multirow[t]{2}{*}{ Producción de capítulos de libros } & Hombres & $1.75(1.055)$ & \multirow{2}{*}{0.476} & \multirow{2}{*}{0.479} \\
\hline & Mujeres & $1.31(1.974)$ & & \\
\hline \multirow[t]{2}{*}{ Producción de artículos científicos } & Hombres & $4.50(3.477)$ & \multirow{2}{*}{0.330} & \multirow{2}{*}{0.858} \\
\hline & Mujeres & $4.23(3.919)$ & & \\
\hline \multirow[t]{2}{*}{ Producción de ponencias } & Hombres & $7.25(3.108)$ & \multirow{2}{*}{0.007} & \multirow{2}{*}{0.936} \\
\hline & Mujeres & $7.15(2.853)$ & & \\
\hline \multirow[t]{2}{*}{ Edad } & Hombres & $45.67(8.281)$ & \multirow{2}{*}{0.258} & \multirow{2}{*}{0.617} \\
\hline & Mujeres & $43.85(5.548)$ & & \\
\hline
\end{tabular}

Se realizó el análisis de regresión múltiple. Como variables predictoras estuvieron los años de experiencia docente, las horas dedicadas a la investigación, la edad y el género.El 52,8\% de los cambios en la producción científica de los docentes se debe a los cambios de las horas dedicadas a la investigación y de los años de experiencia docente. Esto significa que más de la mitad de aumento de la producción científica de los docentes es debida al aumento de las horas dedicadas a la investigación y de los años de experiencia docente. Puede observarse en la Tabla 3 que las variables horas dedicadas a la investigación y años de experiencia docente resultaron significativas (Intervalo de confianza al 95\%: 0.575; 1.738 y 0.914; 2.832 ); las variables edad y 
género no fueron significativas. El modelo de regresión tuvo un buen ajuste $(F=3.500 ; p=0.025)$. No existió colinealidad entre las variables estudiadas.

Con estos resultados se puede afirmar que al aumentar las horas dedicadas a la investigación aumenta la producción científica, cuando los docentes tienen los mismos años de experiencia docente; además, en la medida en que aumentan los años de experiencia docente, aumenta la producción científica, cuando tienen la misma cantidad de horas dedicadas a la investigación. En el aumento o disminución de la producción científica de los docentes no influye ni la edad ni el género.

Tabla 3: Análisis de regresión de la producción científica sobre el resto de las variables del estudio. * $p<0.01$

\begin{tabular}{|l|l|l|l|l|l|}
\hline \multirow{2}{*}{ Variables } & \multirow{2}{*}{$\beta$} & \multirow{2}{*}{ Estadígrafo $t$} & \multirow{2}{*}{$p$} & \multicolumn{2}{c|}{ Índices de colinealidad } \\
\cline { 5 - 6 } & & & & Tolerancia & FIV \\
\hline Horas dedicadas a la investigación & 1.207 & 3.167 & $0.005^{*}$ & 0.575 & 1.738 \\
\hline Años de experiencia docente & 0.093 & 3.235 & $0.006^{*}$ & 0.581 & 1.649 \\
\hline Edad & 0.103 & 0.532 & 0.660 & 0.721 & 1.387 \\
\hline Género & 2.628 & 0.903 & 0.377 & 0.949 & 1.054 \\
\hline
\end{tabular}

\section{DISCUSIÓN}

Realizar una comparación longitudinal de la producción científica de los docentes de la FCEHyT en relación a su experiencia en la docencia y el total de horas dedicadas a la investigación, como punto de partida para establecer mecanismos que permitan aumentar la calidad y la cantidad de publicaciones de cara a los procesos de evaluación y acreditación universitaria ha permitido establecer inferencias sobre las necesidades del personal académico.

Como es bien conocido, en el Ecuador los académicos tienen como exigencia actual la publicación constante, y la presión para hacerlo se ha venido incrementando en los últimos años (Muñoz y Gutiérrez, 2015). En el reto de responder a estos requerimientos se han encontrado con serias dificultades, una de ellas la falta de tiempo, debido a las crecientes responsabilidades dentro de la docencia, la vinculación con la sociedad (Polaino y Romillo, 2017) y las actividades administrativas. Otra dificultad está asociada a la falta de recursos como consecuencia de los presupuestos reducidos de las universidades. Por otra parte, la poca experiencia en la escritura académica representa un desafío, especialmente para el nuevo personal con una cultura de investigación emergente. Al parecer, estos serios inconvenientes afectan a un número considerable del claustro docente; amén de las esporádicas capacitaciones que se han realizado para el desarrollo de competencias, estrategias personales y habilidades para el incremento de la producción científica en los docentes de la FCEHyT.

Esta investigación dilucidó un factor preponderante para la producción académica del personal docente de la FCEHyT, el cual radica en la experiencia académica universitaria. En ese sentido, los docentes que han realizado el mayor número de obras científicas son aquellos que han laborado en un promedio de seis a diez años en las IES pero con una dedicación total a tiempo completo y con un número importante de horas asignadas para la investigación; es decir, existe un cambio que bordea el $52.8 \%$ de incremento de la producción científica en base a las horas asignadas. En este punto, es oportuno destacar el compromiso de los docentes con mayor experiencia al llevar a la reflexión sobre su práctica educativa y los procesos de investigación encaminados a la búsqueda de condiciones transformadoras del rol educativo. Se cree que ello obedece a que el docente con trayectoria académica dilatada que se encuentra inmerso en actividades de investigación tiende a asumir de forma crítica el contenido de sus asignaturas y a trabajar de forma interdisciplinaria, para que su experiencia sea compartida en revistas indexadas y de alto impacto. Los docentes con mayor experiencia han afrontado el reto de la universidad del siglo XXI para el Ecuador, lo que les ha permitido dominar ciertos aspectos tradicionales de la docencia, integrándolos exitosamente con la investigación y por ende acrecentando su producción científica. Para estos docentes, el hábito de la publicación constante contribuye a su desarrollo profesional y personal e influye positivamente en su labor académica.

El personal docente con mayor tiempo de servicio en la FCEHyT ha iniciado además estudios de postgrado, algunos de ellos han alcanzado el grado de Doctor en Ciencias (PhD). Esta es una razón que se adiciona a su incursión en actividades de investigación, lo cual les ha permitido generar una cultura de divulgación científica, involucrando a los profesores con menor experiencia en el trabajo colaborativo y el desarrollo de habilidades de investigación. Se recomienda en este sentido que aquellos docentes con más habilidades para el trabajo colaborativo, mayor tiempo de servicio y formación de postgrado trastoquen el sistema educativo 
tradicional caracterizado por el trabajo individualizado; y si bien es cierto que no todo el personal longevo en las IES tiende a realizar procesos de escritura académica, se aproveche la predisposición existente en algunos de los más experimentados a producir científicamente, con la ayuda de un equipo de investigación, que participe en un ejercicio constante de redacción científica.

Es de resaltar que después de realizar el análisis multivariado de varianza con la variable sexo, se evidenció que tanto hombres como mujeres mantienen una producción científica homogénea. Este resultado desmitifica a la mujer como figura de segundo plano en el orden de la publicación científica, a pesar de ciertas dificultades que afrontan las mujeres para consolidarse dentro del ámbito educativo; pues las mujeres se ven obligadas a duplicar el esfuerzo para lograr un trato igualitario (Ibarra y Llanos, 2016); al parecer algunos de los inconvenientes se relacionan con la cultura organizacional de las IES frente a la construcción de la igualdad de género en el ámbito cultural y académico. En esta investigación ha quedado claro que si bien en el Reglamento de Carrera y Escalafón del Profesor e Investigador del Sistema de Educación Superior del Ecuador, aprobado por el Consejo de Educación Superior (CES), se establece el tipo de personal académico de las IES y el tiempo de dedicación para las labores de docencia, investigación, vinculación y gestión académica, principalmente han condicionado la producción científica dos factores: la antigüedad en la docencia universitaria, y, fundamentalmente, la cantidad de horas asignadas a las actividades de investigación.

Después de establecer la correlación longitudinal entre experiencia docente y carga horaria para la investigación de $r=0.651$ equivalente a $p<0.01$, existe una tendencia a la producción académica con una correlación de $r=0.412$ equivalente a $p<0.05$ que se subyuga al tiempo de dedicación para el efecto. El personal académico con un mayor número de publicaciones durante los últimos cuatro años en la Facultad tiene alrededor de 15 y 30 horas semanales asignadas para desarrollar proyectos de investigación, publicación de libros, capítulos de libros, artículos originales y de revisión, lo cual dista significativamente de aquellos docentes con mayor carga académica y administrativa. (Toala, Meza y Mejía, 2017)

El personal académico con un número menor de horas dedicadas a la investigación no ha desarrollado ciertas habilidades investigativas producto del limitado tiempo que tienen para realizarlo, aunado a la falta de motivación y la escasa capacitación recibida. Por otra parte, al no pertenecer a grupos multidisciplinares de investigación, se ha limitado preocupantemente la producción de resultados científicos en revistas de impacto, o redacción de capítulos de libros (Weissmann, 2014). Este personal académico, con ínfimas horas para la investigación, ha reducido su espacio de producción de conocimiento a eventos científicos en los cuales presentan ponencias o resúmenes de investigaciones. No obstante, hay que destacar que los docentes tratan de cubrir los gastos que conlleva este proceso con la escasa colaboración de los estamentos universitarios debido a limitaciones presupuestarias (Cappellacci et al., 2015).

Es preciso recalcar que la producción científica de los docentes universitarios se encuentra condicionada por el número de horas de investigación asignadas (Lamarra y Coppola, 2016), la actualización continua y el desarrollo de habilidades para la escritura científica. Por tal motivo, es necesaria la planificación de eventos que permitan potenciar las cualidades investigativas del personal académico, el manejo de instrumentos, acervo bibliográfico, recursos metodológicos, técnicos para el incremento de publicaciones de impacto. (Barbón et al., 2017) Además, el personal docente con mayor carga horaria para actividades de investigación debe comprometerse cada vez más a gestionar grupos o equipos de investigación (Murguía, et al., 2013), que se nutran del personal académico con menor experticia para la producción científica. Esto permitirá dotar de nueva información empírica para la interpretación, análisis y reflexión de hechos que han sido comprobados en la práctica y con la consiguiente publicación de datos relevantes. En ese sentido, la publicación de estos resultados, se convierte en la principal vía para compartir con otros investigadores el conocimiento obtenido (López, 2013). Por otra parte, es de preferencia de la comunidad científica la producción de artículos de impacto sobre la elaboración de libros, capítulos de libros, ponencias o producción regional, en vista de que las publicaciones originales están sujetas al debate llevado a cabo por la comunidad científica, posicionando al investigador como referente en determinada área del conocimiento.

Una de las limitaciones del presente estudio es la definición del número de horas específicas de cada investigador, que se basa en los resultados obtenidos de la producción obtenida en los años 2014, 2015, 2016 y 2017 de los docentes con mayor y menor número de publicaciones. Por otro lado, probablemente el total de la muestra es reducido, al tratarse del personal con horas de investigación y a quienes se aplicó el instrumento diseñado; los docentes que carecían de carga horaria de investigación durante los 2 años en que se realizó el estudio no fueron incluidos en el presente estudio. Por lo tanto, recomendamos que los resultados de este trabajo se valoren a la luz de estas posibles faltas, de ser considerados como insumo para la toma de decisiones y la generación de políticas intra-universitarias respecto a la asignación de horas para la investigación y la capacitación docente. Otro aspecto que puede haber constituido un sesgo es que la categoría producción de capítulos de libros, específicamente en el año 2014 puede haber incluido algún 
registro que no se ajuste al requisito actual de revisión por pares académicos, lo cual puede haber dado lugar a una sobrestimación de los resultados entre los docentes con mayor número de publicaciones. Sin embargo, se decidió incluir los datos correspondientes al 2014, por ser el año en que inició el despegue de la producción científica en la facultad.

\section{CONCLUSIONES}

En suma, según los resultados de este estudio, y de su discusión y análisis se pueden extraer las siguientes conclusiones:

a) El perfeccionamiento de las capacidades investigativas en los docentes universitarios permite, a corto, mediano y largo plazo, resolver los problemas profesionales desde un enfoque científico e investigativo. Este proceso ha sido inducido en el marco de los profundos cambios que lleva actualmente a cabo la Universidad Nacional de Chimborazo, en la pesquisa de la calidad y excelencia en la Educación Superior ecuatoriana.

b) El principal aporte de la investigación realizada estriba en que la misma, al explorar la capacidad predictiva del género, la edad, las horas dedicadas a la investigación y la experiencia docente sobre la producción científica, ha favorecido la identificación de los docentes cuya productividad científica es evidente, de manera que se puedan adoptar medidas de apoyo al resto del personal docente.

c) Por último, este trabajo constituye un primer acercamiento a trazar el perfil general del investigador idóneo, así como de los factores que afectan a este perfil, de modo que se puedan emprender iniciativas, en el menor tiempo posible, como política del mejoramiento de la calidad y excelencia de la investigación en la FCEHyT y en la universidad en general. En este sentido, se constató que el personal académico con un mayor número de publicaciones durante los últimos cuatro años en la Facultad, tiene alrededor de 15 y 30 horas semanales asignadas para el desarrollo de proyectos de investigación, publicación de libros, capítulos de libros y artículos científicos. Este hallazgo podría ser una interesante línea a seguir por una futura investigación.

\section{AGRADECIMIENTOS}

Los autores agradecen el apoyo de la Universidad Nacional de Chimborazo y en particular del Vicerrectorado de Posgrado e Investigación y de la Facultad de Ciencias de la Educación, Humanas y Tecnologías.

\section{REFERENCIAS}

Abramo, G., C.A. D'Angelo y F. Rosati, Career advancement and scientific performance in universities, Scientometrics, 98(2), 891-907 (2014)

Álvarez, L. y J. Juncosa, La evaluación y acreditación de la educación superior ecuatoriana y la producción editorial, Universitas, XII (21), 163-181 (2014)

Aranguren, M., S. Franco, R. Horta y L. Silveira, Retos y aprendizajes de institutos de investigación transformadora, Journal of technology management \& innovation, 11(1), 69-79 (2016)

Barbón, O. G. Á. del R. Calderón y otros tres autores, Algunos problemas de docentes universitarios en la elaboración de artículos científicos, Actualidades Investigativas en Educación, 17(1) (2017)

Cabezas, Á., E. Jiménez y E.D. López ¿Existe relación entre el tamaño del grupo de investigación y su rendimiento científico? Estudio de caso de una universidad española, Revista española de documentación científica, $36(2), 006$ (2013)

Cappellacci, I., B. Briscioli y otros cuatro autores, Institucionalizar la función de investigación en la formación docente, Educación, Formación e Investigación, 1(1) (2015)

Farcas, D., S. Bernardes y M. Matos, The research-teaching nexus from the Portuguese academics' perspective: a qualitative case study in a school of social sciences and humanities, Higher Education, 74(2), 239-258 (2017)

Franzoni, C. y H. Sauermann, Crowd science: The organization of scientific research in open collaborative projects, https://doi.org/10.1016/j.respol.2013.07.005, Research Policy, 43(1), 1-20 (2014)

Herrero, L., The future of Spanish public Universities: The challenges of the excellence debate. Encounters on Education, [S.I.],16, 146-164 (2015)

Ibarra, M. E. y G.C. Llanos, Género y educación superior. Un análisis de la participación de las mujeres como profesoras en la Universidad del Valle, La manzana de la discordia, 4(1), 73-92 (2016)

Jiménez, M. B. y R.B. Medina, Incidencia de la motivación en la producción científica institucional Impact of motivation in institutional scientific production, Revista Retos, 11(1), 65-87 (2016)

Kemmis, S., R. McTaggart y R. Nixon, Introducing Critical Participatory Action Research. In The Action Research Planner, Springer Singapore, 1-31 (2014) 
Lamarra, N.F. y N. Coppola, La Evaluación de la Docencia Universitaria en Argentina. Situación, Problemas y Perspectivas, Revista Iberoamericana de Evaluación Educativa, 1(3e) (2016)

Muñoz, P. A. y M.P. Gutiérrez, Análisis de la producción y de la visibilidad científica de Ecuador en el contexto andino (2000-2013), El profesional de la información, 24(5), 577-586 (2015)

Murguía, R., Y. Villa, E. González y J. Martínez, Metodología para la gestión de la calidad de los procesos en instituciones de educación superior, Sistemas \& Telemática, 11(26), 47-58 (2013)

Osborne, J., Teaching scientific practices: Meeting the challenge of change, Journal of Science Teacher Education, 25(2), 177-196 (2014)

Parra, M.C., Las políticas de ciencia y tecnología en Venezuela y su Impacto en el sistema universitario en el estado Zulia, Interciencia, 419-426 (2007)

Polaino, C.J. y A. de J. Romillo, Vinculación con la Sociedad en la Universidad de Otavalo, Ecuador, https://dx.doi.org/10.4067/S0718-50062017000300004, Formación Universitaria, 10(3), 21-30 (2017)

Rabossi, M., The agency costs in university institutions: an analytical model for its evaluation, Education policy analysis archives, 22, $102(2014)$

Rey, J., B. Garzón y M.J. Martín, Consolidación y cohesión de los grupos de investigación del CSIC y su influencia sobre la actividad investigadora y el rendimiento de sus componentes: Área de Ciencia y Tecnologías Químicas, Informe CSIC (2010)

Romero, J. y J.M. Pastor, Las universidades españolas bajo la influencia de los rankings, Regional and Sectoral Economics Studies, 12(3), 105-126 (2012)

Rossi, F. y A. Rosli, Indicators of university-industry knowledge transfer performance and their implications for universities: evidence from the United Kingdom, http://dx.doi.org/10.1080/03075079.2014.914914, Studies in Higher Education, 40(10), 1970-1991 (2015)

Simonneaux, L., Questions socialement vives and socio-scientific issues: New trends of research to meet the training needs of postmodern society. In Topics and trends in current science education, Springer Netherlands, 37-54 (2014)

Schubert, T. y G. Yang, Institutional change and the optimal size of universitie, https://doi.org/10.1007/s11192-016-20151, Scientometrics, 108(3), 1129-1153 (2016)

Toala, G., D. Meza y G. Mejía, Modelo de gestión del conocimiento y capital intelectual de los docentes universitarios, Revista Publicando, 4(11), 270-286 (2017)

Weissmann, P., Relación entre dedicación horaria y desempeño docente. Entramados: educación y sociedad, 1(1), 267273 (2014) 